\title{
A Chebyshev-Gauss-Radau Scheme For Nonlinear Hyperbolic System Of First Order
}

\author{
E. H. Doha ${ }^{1}$, A. H. Bhrawy ${ }^{2,3}$, R. M. Hafez ${ }^{4}$ and M. A. Abdelkawy ${ }^{3, *}$ \\ ${ }^{1}$ Department of Mathematics, Faculty of Science, Cairo University, Giza, Egypt \\ 2 Department of Mathematics, Faculty of Science, King Abdulaziz University, Jeddah, Saudi Arabia \\ ${ }^{3}$ Department of Mathematics, Faculty of Science, Beni-Suef University, Beni-Suef, Egypt \\ ${ }^{4}$ Department of Basic Science, Institute of Information Technology, Modern Academy, Cairo, Egypt
}

Received: 15 Mar. 2013, Revised: 15 Jul. 2013, Accepted: 17 Jul. 2013

Published online: 1 Mar. 2014

\begin{abstract}
A numerical approximation of the initial-boundary system of nonlinear hyperbolic equations based on spectral collocation method is presented in this article. A Chebyshev-Gauss-Radau collocation (C-GR-C) method in combination with the implicit RungeKutta scheme are employed to obtain highly accurate approximations to the mentioned problem. The collocation points are the Chebyshev interpolation nodes. This approach reduces this problem to solve system of nonlinear ordinary differential equations which are far easier to be solved. Indeed, by selecting a limited number of collocation nodes, we obtain an accurate results. The numerical examples demonstrate the accuracy, efficiency, and versatility of the method.
\end{abstract}

Keywords: System of nonlinear hyperbolic equations; Collocation method; Chebyshev-Gauss-Radau quadrature; Implicit RungeKutta method

\section{Introduction}

In the last decades, partial differential hyperbolic system has a wide range of physical applications such as viscoelastic phenomena [1]-[6] and plants control systems' models [7]. Hyperbolic systems have been studied in many articles analytically and numerically. Finite element methods have been used in [8]-[11] to numerically solve different examples of hyperbolic system. While, finite volume method base on curved manifold wave propagation algorithm [12], diagonally implicit-explicit Runge-Kutta time-marching algorithm [13], ADER scheme [14] have been used in the study of numerical solution for hyperbolic system types. Numerical solution based on improved predictor-corrector method has been discussed in [15] for solving hybrid fuzzy differential equations. Moreover, many researchers have used spectral methods for solving many types of hyperbolic models, see for examples [16][20]. Also, many analytical method have been introduced to obtain exact solutions for hyperbolic models, see for examples [21,22].
One of the more accurate numerical methods is spectral method. Spectral methods have emerged as a powerful techniques used in applied mathematics and scientific computing to numerically solve linear and nonlinear differential equations [23]-[26], integral equations [27]-[30], integro-differential equations [31]-[34], function approximation and variational problems [35]-[38]. The main idea of spectral methods is to put the solution of the problem as a sum of certain basis functions and then to choose the coefficients in the sum in order to minimize the difference between the exact solution and approximate one as well as possible. The choice of test functions divided spectral methods into three well-known types, namely, the Galerkin [39]-[42], tau [43]-[45] and collocation methods [46]-[48]. Spectral collocation method has an exponential convergence rate, which is very useful in providing highly accurate solutions to nonlinear differential equations even using a small number of grids. In contrast, finite-difference and finite-element methods yield only algebraic convergence rates. Also it has became increasingly popular for solving fractional differential equations [49]-[51].

\footnotetext{
*Corresponding author e-mail: melkawy@yahoo.com
} 
The widely applicable, efficiency, and high accuracy are the more advantages of the collocation method. Due to these advantages of spectral collocation method when compared with other numerical methods, many studies about this method have been introduced. In this way, Chebyshev collocation methods were proposed for solving linear and nonlinear ordinary differential equations [52] and fractional order differential equations [53]. Meanwhile, numerical treatments based on Chebyshev collocation method for some partial differential equations are introduced in $[54,55,56]$. Moreover, Chebyshev collocation methods were introduced for numerically solving integral equations [57, $58]$ and integro-differential equations [59,60].

In the present paper, we numerically solve two hyperbolic systems of first order which can be formulated as the propagation of shallow water waves and the Brusselator model of the chemical reaction-diffusion models. In fact, we treat with these problems in two steps. The first one, C-GR-C method is used to reduce the hyperbolic system of first order to a system of first order ordinary differential equations in time. Implicit Runge-Kutta scheme is employed to obtain numerical solution for the mentioned system of first order ordinary differential equations in time. To the best of our knowledge, there are no results on C-GR-C method for solving the hyperbolic system of first order which we will be discussed in this paper.

The outline of this paper is arranged as follows. We present some properties of Chebyshev polynomials in the next section. Section 3 is devoted to develop the C-GR-C method for solving the hyperbolic system of first order. Some numerical examples are proposed in Section 4 to show the accuracy of our method. In the last section, we present some observations and conclusions.

\section{Some properties of Chebyshev polynomials}

The well-known Chebyshev polynomials are defined on the interval $[-1,1]$ and can be determined with the aid of the following recurrence formula:

$$
T_{k+1}(x)=2 x T_{k}(x)-T_{k-1}(x), \quad k=1,2, \cdots,
$$

where $T_{0}(x)=1$ and $T_{1}(x)=x$. The Chebyshev polynomials are satisfying the following relations

$$
T_{k}( \pm 1)=( \pm 1)^{k}
$$

Let $w(x)=\frac{1}{\sqrt{1-x^{2}}}$, then we define the weighted space $L_{w}^{2}$ as usual. The inner product and the norm of $L_{w}^{2}$ with respect to the weight function are defined as follows:

$$
(u, v)_{w}=\int_{-1}^{1} u(x) v(x) w(x) d x, \quad\|u\|_{w}=(u, u)_{w}^{\frac{1}{2}} .
$$

The set of Chebyshev polynomials forms a complete $L_{w^{-}}^{2}$ orthogonal system, and

$$
\left\|T_{k}\right\|_{w}=h_{k}=\left\{\begin{array}{l}
\frac{C_{k}}{2} \pi, \quad k=j, \quad C_{0}=2, \quad C_{k}=1, \quad k \geq 1 . \\
0, \quad k \neq j,
\end{array}\right.
$$

\section{Chebyshev spectral collocation method}

The main objective of this section is to present the C-GR-C method to numerically solve the system of nonlinear hyperbolic equations.

Firstly, we are interested in using the C-GR-C method to transform the following partial differential equations (PDEs) into system of ordinary differential equations (SODEs)

$$
\begin{gathered}
\frac{\partial u(y, t)}{\partial t}+\frac{\partial u(y, t)}{\partial y}+\eta u(y, t) v(y, t)=0, \\
\frac{\partial v(y, t)}{\partial t}-\frac{\partial v(y, t)}{\partial y}+\eta u(y, t) v(y, t)=0, \\
(y, t) \in[A, B] \times[0, T],
\end{gathered}
$$

where $\eta$ is a constant, with the boundary-initial conditions

$$
\begin{array}{lll}
u(A, t)=g_{1}(t), & v(A, t)=g_{2}(t), & t \in[0, T], \\
u(y, 0)=f_{1}(y), & v(y, 0)=f_{2}(y), & y \in[A, B] .
\end{array}
$$

Now, suppose the change of variables $x=\frac{2}{B-A} y+\frac{A+B}{A-B}, \quad u(y, t)=r(x, t), \quad v(y, t)=s(x, t)$, which will be used to transform problem (4)-(5) into the following system in the classical interval, $[-1,1]$, for the space variable, to directly implement the collocation method based on Chebyshev family defined on $[-1,1]$,

$$
\begin{gathered}
\frac{\partial r(x, t)}{\partial t}+\left(\frac{2}{B-A}\right) \frac{\partial r(x, t)}{\partial x}+\eta r(x, t) s(x, t)=0, \\
\frac{\partial s(x, t)}{\partial t}-\left(\frac{2}{B-A}\right) \frac{\partial s(x, t)}{\partial x}+\eta r(x, t) s(x, t)=0, \\
(x, t) \in[-1,1] \times[0, T],
\end{gathered}
$$

with the boundary-initial conditions

$$
\begin{aligned}
& r(-1, t)=g_{3}(t), \quad s(-1, t)=g_{4}(t), \quad t \in[0, t] \\
& r(x, 0)=f_{3}(x), \quad s(x, 0)=f_{4}(x), \quad x \in[-1,1] .
\end{aligned}
$$

Now, we outline the main steps of applying the C-GR-C method for solving system of nonlinear hyperbolic equations. In the Chebyshev collocation method, the approximate solutions can be expressed as a truncated Chebyshev series:

$$
\begin{aligned}
& r(x, t)=\sum_{j=0}^{N} a_{j}(t) T_{j}(x), \\
& s(x, t)=\sum_{j=0}^{N} b_{j}(t) T_{j}(x),
\end{aligned}
$$


and in virtue of (2)-(3), we deduce that

$$
\begin{aligned}
& a_{j}(t)=\frac{1}{h_{j}} \int_{-1}^{1} r(x, t) w(x) T_{j}(x) d x, \\
& b_{j}(t)=\frac{1}{h_{j}} \int_{-1}^{1} s(x, t) w(x) T_{j}(x) d x .
\end{aligned}
$$

To evaluate the previous integrals accurately, we present the Chebyshev-Gauss-Radau quadrature. For any $\phi \in S_{2 N}(-1,1)$,

$$
\int_{-1}^{1} w(x) \phi(x) d x=\sum_{j=0}^{N} \varpi_{N, j} \phi\left(x_{N, j}\right),
$$

where $S_{N}(-1,1)$ is the set of polynomials of degree less than or equal to $N$ and $x_{N, j}(0 \leq j \leq N)$ and $\varpi_{N, j}$ $(0 \leq j \leq N)$ are the nodes and the corresponding Christoffel numbers of the Chebyshev-Gauss-Radau quadrature formula on the interval $[-1,1]$, respectively. In accordance to (2), the coefficients $a_{j}(t)$ in terms of the solution at the collocation points can be approximated by

$$
\begin{aligned}
& a_{j}(t)=\frac{1}{h_{j}} \sum_{i=0}^{N} T_{j}\left(x_{N, i}\right) \varpi_{N, i} r\left(x_{N, i}, t\right), \\
& b_{j}(t)=\frac{1}{h_{j}} \sum_{i=0}^{N} T_{j}\left(x_{N, i}\right) \varpi_{N, i} s\left(x_{N, i}, t\right) .
\end{aligned}
$$

Therefore, (8) can be rewritten as

$$
\begin{aligned}
& r(x, t)=\sum_{i=0}^{N}\left(\sum_{j=0}^{N} \frac{1}{h_{j}} T_{j}\left(x_{N, i}\right) T_{j}(x) \varpi_{N, i}\right) r\left(x_{N, i}, t\right), \\
& s(x, t)=\sum_{i=0}^{N}\left(\sum_{j=0}^{N} \frac{1}{h_{j}} T_{j}\left(x_{N, i}\right) T_{j}(x) \varpi_{N, i}\right) s\left(x_{N, i}, t\right) .
\end{aligned}
$$

Furthermore, if we differentiate (12) once, and evaluate it at all C-GR-C points, it is easy to compute the first spatial partial derivative in terms of the values at these collocation points as

$$
\begin{aligned}
& r_{x}\left(x_{N, n}, t\right)=\sum_{i=0}^{N} A_{n i} r\left(x_{N, i}, t\right), \\
& s_{x}\left(x_{N, n}, t\right)=\sum_{i=0}^{N} B_{n i} s\left(x_{N, i}, t\right), \quad n=0,1, \cdots, N,
\end{aligned}
$$

where

$$
\begin{aligned}
A_{n i} & =\sum_{j=0}^{N} \frac{1}{h_{j}} T_{j}\left(x_{N, i}\right) \partial_{x} T_{j}\left(x_{N, n}\right) \varpi_{N, i}, \\
B_{n i} & =\sum_{j=0}^{N} \frac{1}{h_{j}} T_{j}\left(x_{N, i}\right) \partial_{x x} T_{j}\left(x_{N, n}\right) \varpi_{N, i} .
\end{aligned}
$$

In the proposed C-GR-C method the residual of (6) is set to zero at $N$ of Chebyshev-Gauss-Radau points. Moreover, the boundary conditions (7) will be enforced at the collocation point -1 . Therefore, adopting (12)-(14), enable one to write (6)-(7) in the form:

$$
\begin{aligned}
& \dot{\mathrm{r}}_{n}(t)=-\left(\frac{2}{B-A}\right) \sum_{i=0}^{N} A_{n i} r_{i}(t)-\eta r_{n}(t) s_{n}(t), \\
& \dot{\mathrm{s}}_{n}(t)=\left(\frac{2}{B-A}\right) \sum_{i=0}^{N} B_{n i} s_{i}(t)-\eta r_{n}(t) s_{n}(t),
\end{aligned}
$$

where

$$
\begin{aligned}
& r_{k}(t)=r\left(x_{N, k}, t\right), \quad s_{k}(t)=s\left(x_{N, k}, t\right), \\
& k=1, \cdots, N, \quad n=1, \cdots, N .
\end{aligned}
$$

This provides a $(2 N)$ system of first order ordinary differential equations in the expansion coefficients $a_{j}(t)$, namely

$$
\begin{aligned}
& \dot{\mathrm{r}}_{n}(t)=-\left(\frac{2}{B-A}\right)\left(\sum_{i=1}^{N} A_{n i} r_{i}(t)+A_{n 0} r_{0}(t)\right)-\eta r_{n}(t) s_{n}(t), \\
& \dot{\mathrm{s}}_{n}(t)=\left(\frac{2}{B-A}\right)\left(\sum_{i=1}^{N} B_{n i} s_{i}(t)+B_{n 0} s_{0}(t)\right)-\eta r_{n}(t) s_{n}(t) .
\end{aligned}
$$

This means that problem (4)-(5) is transformed into the following SODEs

$$
\begin{aligned}
& \dot{\mathrm{r}}_{n}(t)=-\left(\frac{2}{B-A}\right)\left(\sum_{i=1}^{N} A_{n i} r_{i}(t)+A_{n 0} r_{0}(t)\right)-\eta r_{n}(t) s_{n}(t), \\
& \dot{\mathrm{s}}_{n}(t)=\left(\frac{2}{B-A}\right)\left(\sum_{i=1}^{N} B_{n i} s_{i}(t)+B_{n 0} s_{0}(t)\right)-\eta r_{n}(t) s_{n}(t) .
\end{aligned}
$$

subject to the initial values

$$
r_{n}(0)=f_{3}\left(x_{N, n}\right), \quad s_{n}(0)=f_{4}\left(x_{N, n}\right), \quad n=1, \cdots, N
$$

Finally, (17)-(18) can be rewritten into a matrix form of $2 N$ system of first order ODEs with their vectors of initial values:

$$
\begin{aligned}
\dot{\mathbf{w}}(t) & =\mathbf{F}(t, r(t), s(t)), \\
\mathbf{w}(0) & =\mathbf{f}
\end{aligned}
$$

where

$$
\begin{gathered}
\dot{\mathbf{w}}(t)=\left[\dot{\mathrm{r}}_{1}(t), \dot{\mathrm{r}}_{2}(t), \ldots, \dot{\mathrm{r}}_{N}(t), \dot{\mathrm{s}}_{1}(t), \dot{\mathrm{s}}_{2}(t), \ldots, \dot{\mathrm{s}}_{N}(t)\right]^{T} \\
\mathbf{f}=\left[f_{3}\left(x_{N, 1}\right), \ldots, f_{3}\left(x_{N, N}\right), f_{4}\left(x_{N, 1}\right), \ldots, f_{4}\left(x_{N, N}\right)\right]^{T}
\end{gathered}
$$


and

$$
\begin{gathered}
\mathbf{F}(t, u(t))=\left[F_{1}(t, u(t)), F_{2}(t, u(t)), \ldots, F_{N}(t, u(t)),\right. \\
\left.G_{1}(t, u(t)), G_{2}(t, u(t)), \ldots, G_{N}(t, u(t))\right]^{T},
\end{gathered}
$$

where

$$
\begin{aligned}
F_{n}(t, u(t))= & -\left(\frac{2}{B-A}\right)\left(\sum_{i=1}^{N} A_{n i} r_{i}(t)+A_{n 0} r_{0}(t)\right)- \\
& \eta r_{n}(t) s_{n}(t),
\end{aligned}
$$

$G_{n}(t, u(t))=\left(\frac{2}{B-A}\right)\left(\sum_{i=1}^{N} B_{n i} s_{i}(t)+B_{n 0} s_{0}(t)\right)-\eta r_{n}(t) s_{n}(t)$.

The system of first order ODEs (19) can be solved by implicit Runge-Kutta scheme.

\section{Numerical results}

In this section, two examples are considered to show the accuracy of the algorithm presented in the previous section. The results obtained, reveal that the present method is very effective and convenient.

Example 1. We now consider the inhomogeneous nonlinear system

$$
\begin{aligned}
& \frac{\partial u(y, t)}{\partial t}+v(y, t) \frac{\partial u(y, t)}{\partial y}+u(y, t)-1=0 \\
& \frac{\partial v(y, t)}{\partial t}-u(y, t) \frac{\partial v(y, t)}{\partial y}-v(y, t)-1=0 \\
& (y, t) \in[-1,1] \times[0,1]
\end{aligned}
$$

with the boundary-initial conditions

$$
\begin{aligned}
& u(0, t)=e^{-t}, \quad v(0, t)=e^{t} \\
& u(y, 0)=e^{y}, \quad v(y, 0)=e^{-y}
\end{aligned}
$$

The exact solutions of Eqs. (22) are

$$
u(y, t)=e^{y-t}, \quad v(y, t)=e^{-y+t},
$$

The difference between the measured or inferred value of approximate solution and its actual value (absolute error), given by

$$
E_{1}(y, t)=|u(y, t)-\widetilde{u}(y, t)|, \quad E_{2}(y, t)=|v(y, t)-\widetilde{v}(y, t)|,
$$

where $u(y, t)(v(y, t))$ and $\widetilde{u}(y, t)(\widetilde{v}(y, t))$ are the exact solution and the approximate solution at the point $(y, t)$,
Table 1: Absolute errors for problem (22).

\begin{tabular}{|c|c|c|c|c|}
\hline \multirow{2}{*}{$(x, t)$} & \multicolumn{2}{|c|}{$n=8$} & \multicolumn{2}{c|}{$n=10$} \\
\cline { 2 - 5 } & $M_{1}$ & $M_{2}$ & $M_{1}$ & $M_{2}$ \\
\hline$(-1,0.0)$ & $1.99 .10^{-7}$ & $1.99 .10^{-7}$ & $5.50 .10^{-10}$ & $5.50 .10^{-10}$ \\
\hline$(-0.8,0.1)$ & $8.38 .10^{-8}$ & $9.04 .10^{-8}$ & $7.70 .10^{-10}$ & $2.77 .10^{-8}$ \\
\hline$(-0.6,0.2)$ & $6.61 .10^{-8}$ & $1.01 .10^{-7}$ & $4.11 .10^{-8}$ & $4.99 .10^{-8}$ \\
\hline$(-0.4,0.3)$ & $1.60 .10^{-7}$ & $2.79 .10^{-7}$ & $4.04 .10^{-8}$ & $2.85 .10^{-8}$ \\
\hline$(-0.2,0.4)$ & $2.97 .10^{-8}$ & $2.31 .10^{-8}$ & $3.10 .10^{-8}$ & $1.19 .10^{-9}$ \\
\hline$(0.0,0.5)$ & $6.35 .10^{-8}$ & $3.88 .10^{-7}$ & $1.11 .10^{-7}$ & $3.64 .10^{-8}$ \\
\hline$(0.2,0.6)$ & $3.35 .10^{-8}$ & $1.64 .10^{-8}$ & $4.60 .10^{-8}$ & $3.05 .10^{-9}$ \\
\hline$(0.4,0.7)$ & $9.47 .10^{-8}$ & $4.35 .10^{-7}$ & $4.52 .10^{-8}$ & $8.75 .10^{-9}$ \\
\hline$(0.6,0.8)$ & $2.98 .10^{-8}$ & $1.57 .10^{-7}$ & $8.99 .10^{-8}$ & $2.98 .10^{-8}$ \\
\hline$(0.8,0.9)$ & $3.02 .10^{-8}$ & $1.30 .10^{-7}$ & $2.00 .10^{-8}$ & $1.64 .10^{-8}$ \\
\hline$(1.0,1.0)$ & $9.01 .10^{-8}$ & $4.31 .10^{-7}$ & $3.88 .10^{-8}$ & $1.73 .10^{-8}$ \\
\hline
\end{tabular}

Table 2: Maximum absolute errors for problem (22).

\begin{tabular}{|c|c|c|}
\hline$N$ & $M_{1}$ & $M_{2}$ \\
\hline 4 & $5.47 \times 10^{-3}$ & $1.93 \times 10^{-2}$ \\
8 & $1.99 \times 10^{-7}$ & $4.31 \times 10^{-7}$ \\
12 & $1.10 \times 10^{-7}$ & $4.77 \times 10^{-8}$ \\
\hline
\end{tabular}

respectively. Moreover, the maximum absolute error is given by

$$
\begin{aligned}
& M_{1}=\operatorname{Max}\left\{E_{1}(y, t): \forall(y, t) \in D \times[0, T]\right\}, \\
& M_{2}=\operatorname{Max}\left\{E_{2}(y, t): \forall(y, t) \in D \times[0, T]\right\} .
\end{aligned}
$$

Absolute errors of $u(y, t)$ and $v(y, t)$ related to (22)-(24) are introduced in Table 1 using C-GR-C method with two choices of $N$. Moreover, maximum absolute errors of $u(y, t)$ and $v(y, t)$ related to (22)-(24) are introduced in Table 2 using C-GL-C method with various choices of $N$, when compared with the exact solution (24). The approximate solutions $\widetilde{u}(x, t)$ and $\widetilde{v}(x, t)$ of problem (22) with $N=12$ are displayed in Figs. 1 and 2, respectively. While, in Figs. 3 and 4, we present the absolute errors $E_{1}(x, t)$ and $E_{2}(x, t)$ with $N=12$. Moreover, we plotted the approximate solutions $\widetilde{u}(x, t)$ and $\widetilde{v}(x, t)$, and the exact solutions $u(x, t)$ and $v(x, t)$ in Figs. 5, 6, 7 and 8 , respectively, with values of parameters listed in their captions. Also wee see that the approximate and exact solutions are coincided for different values of $t$ or $x$.

Example 2. We consider the homogeneous linear system

$$
\begin{aligned}
& \frac{\partial u(y, t)}{\partial t}-\frac{\partial v(y, t)}{\partial y}=0, \\
& \frac{\partial v(y, t)}{\partial t}+\frac{\partial u(y, t)}{\partial y}=0, \quad(y, t) \in[-3,3] \times[0,1]
\end{aligned}
$$

with the boundary-initial conditions

$$
\begin{aligned}
& u(0, t)=\cosh (t)+\sinh (t), \quad v(0, t)=\cosh (t)-\sinh (t), \\
& u(y, 0)=e^{y}, \quad v(y, 0)=e^{-y}
\end{aligned}
$$




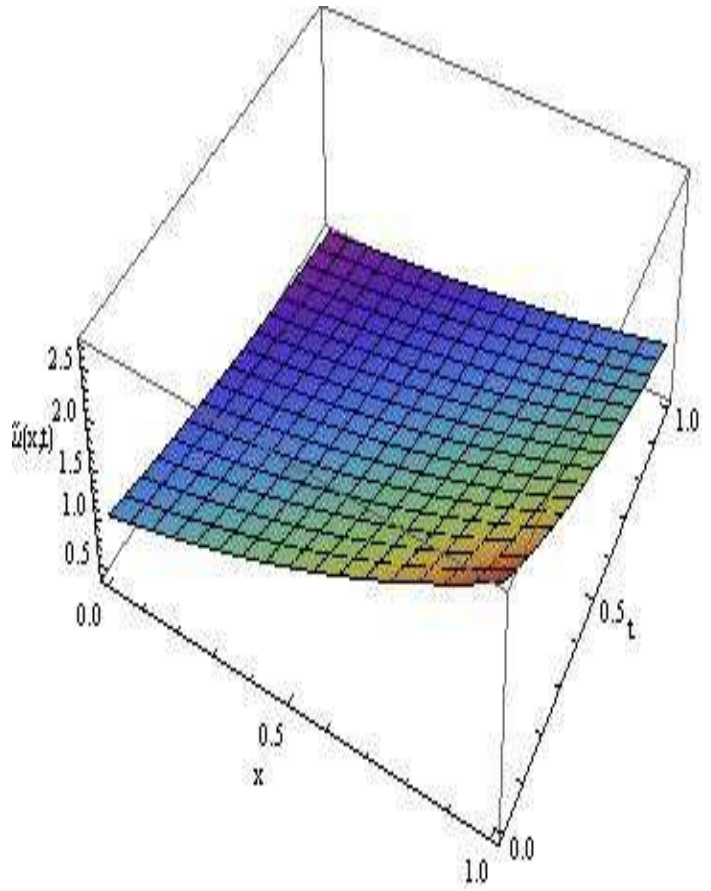

Fig. 1: The approximate solution $\widetilde{u}(x, t)$ for example 1 at $N=12$.

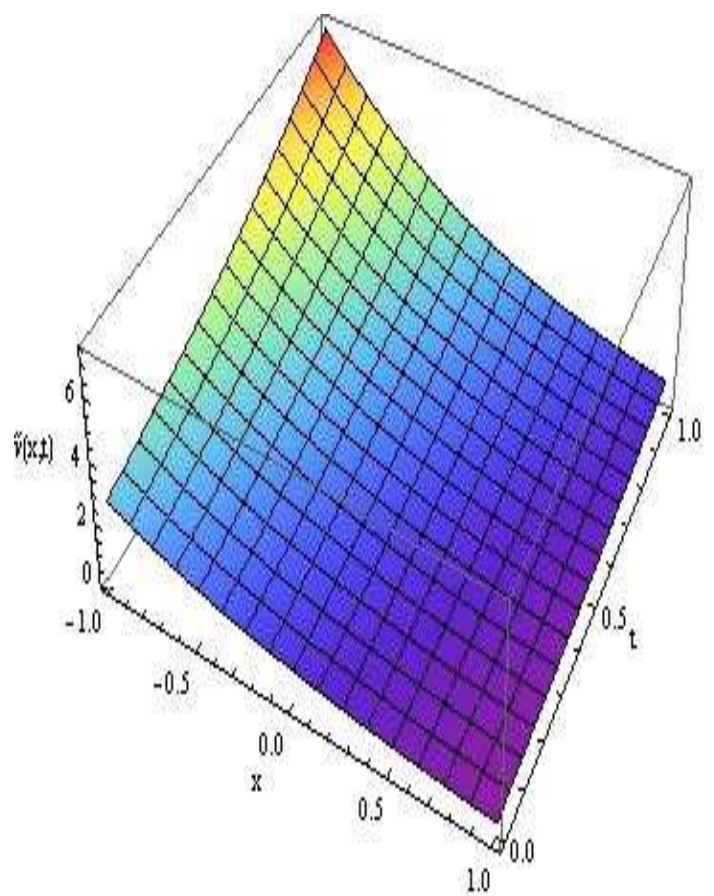

Fig. 2: The approximate solution $\widetilde{v}(x, t)$ for example 1 at $N=12$.

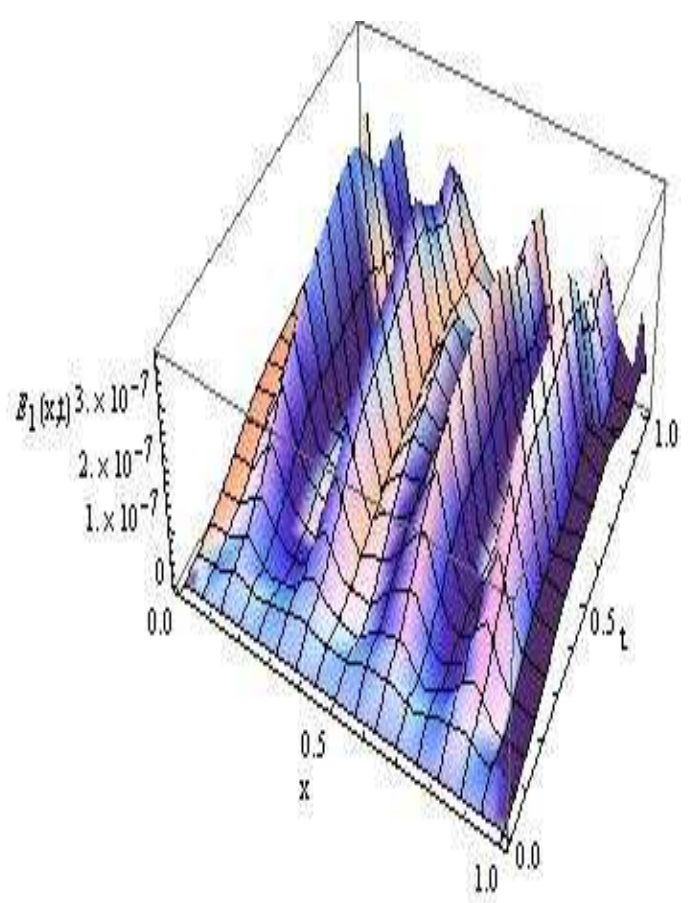

Fig. 3: The absolute error $E_{1}(x, t)$ for example 1 at $N=12$.

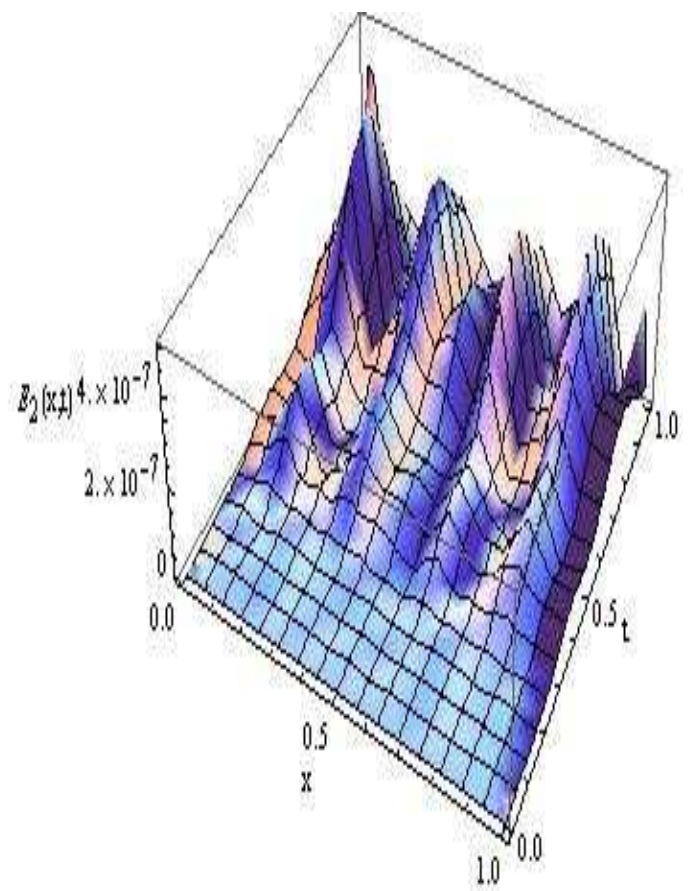

Fig. 4: The absolute error $E_{2}(x, t)$ for example 1 at $N=12$. 


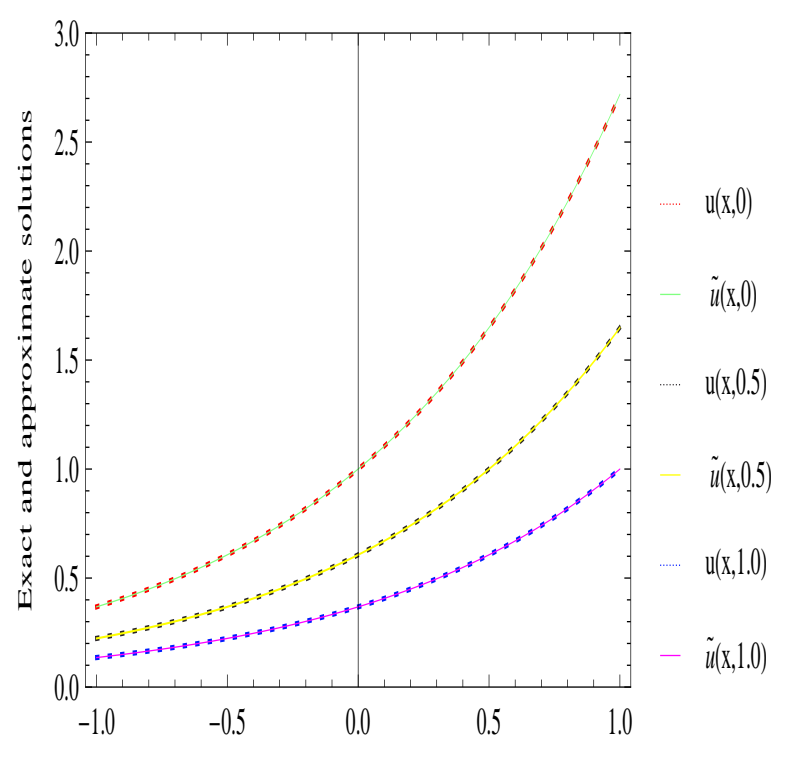

$X$

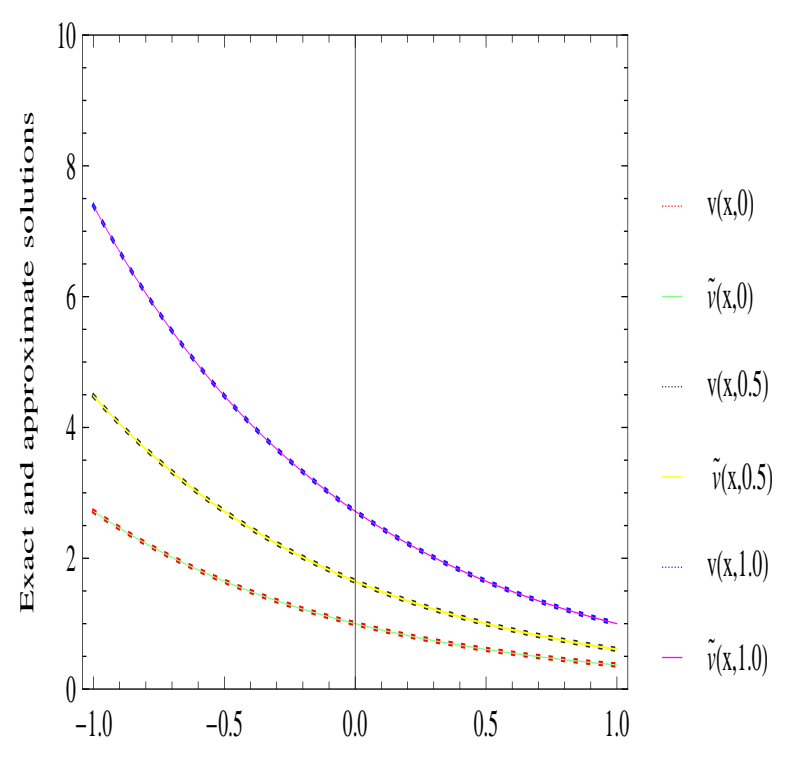

X

Fig. 5: The approximate solution $\widetilde{u}(x, t)$ and the exact solution $u(x, t)$ for $t=0.0,0.5$ and 1.0 for example 1 at $N=12$.

Fig. 7: The approximate solution $\widetilde{v}(x, t)$ and the exact solution $v(x, t)$ for $t=0.0,0.5$ and 1.0 for example 1 at $N=12$.
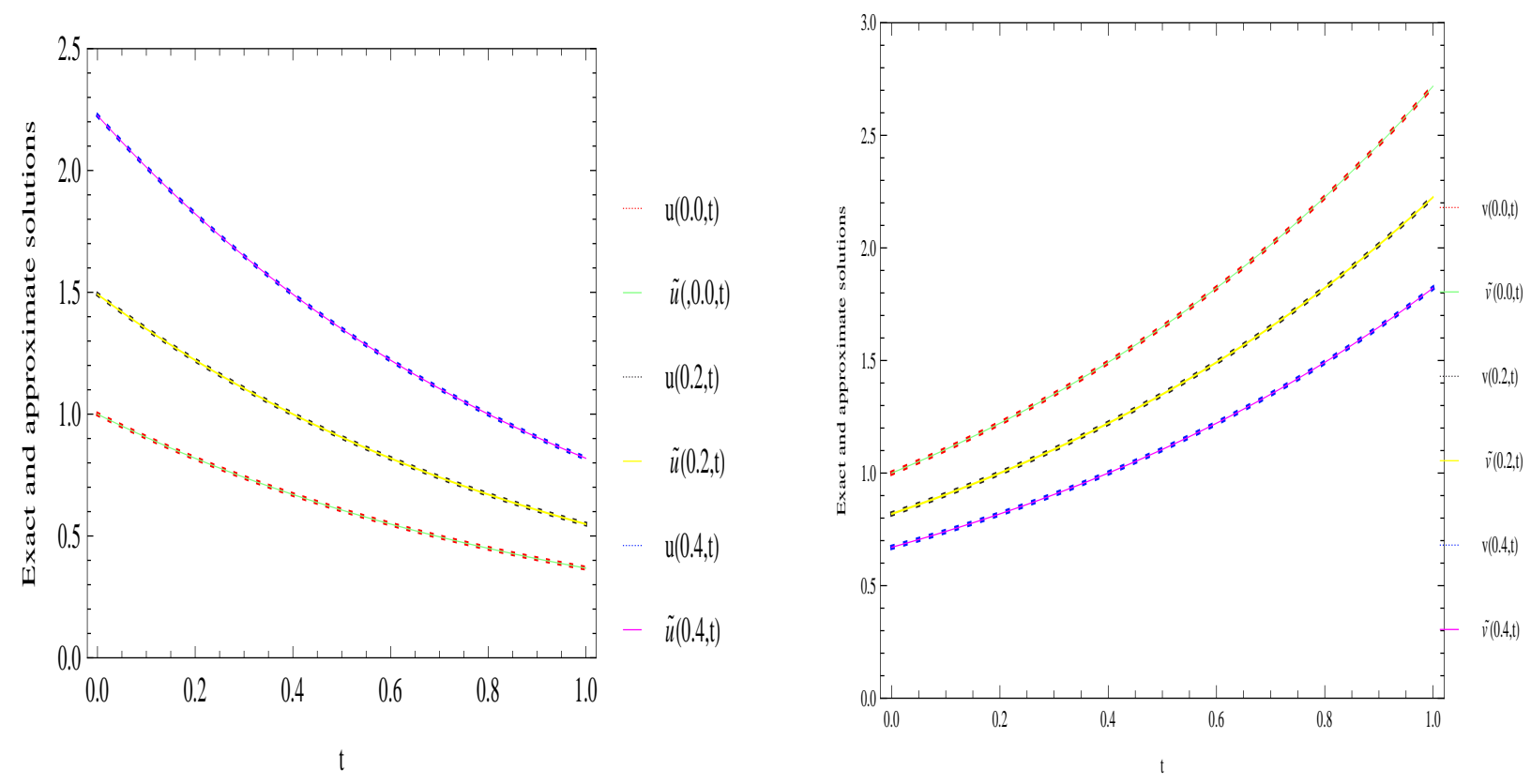

Fig. 6: The approximate solution $\widetilde{u}(x, t)$ and the exact solution $u(x, t)$ for $x=0.0,0.2$ and 0.4 for example 1 at $N=12$.

Fig. 8: The approximate solution $\widetilde{v}(x, t)$ and the exact solution $v(x, t)$ for $x=0.0,0.2$ and 0.4 for example 1 at $N=12$. 
Table 3: Absolute errors for problem (27).

\begin{tabular}{|c|c|c|c|c|}
\hline \multirow[t]{2}{*}{$(x, t)$} & \multicolumn{2}{|c|}{$n=10$} & \multicolumn{2}{|c|}{$n=12$} \\
\hline & $M_{1}$ & $M_{2}$ & $M_{1}$ & $M_{2}$ \\
\hline$(-3,0.0)$ & $3.89 .10^{-5}$ & $3.89 .10^{-5}$ & $6.43 \cdot 10^{-7}$ & $6.43 \cdot 10^{-7}$ \\
\hline$(-2.5,0.1)$ & $3.40 .10^{-5}$ & $3.50 .10^{-5}$ & $6.40 .10^{-7}$ & $9.25 .10^{-7}$ \\
\hline$(-2,0.2)$ & $2.10 .10^{-5}$ & $1.53 .10^{-5}$ & $5.38 .10^{-7}$ & $2.09 .10^{-7}$ \\
\hline$(-1.5,0.3)$ & $1.21 .10^{-5}$ & $2.86 .10^{-5}$ & $7.86 .10^{-7}$ & $4.36 .10^{-7}$ \\
\hline$(-1,0.4)$ & $3.14 .10^{-5}$ & $4.17 .10^{-5}$ & $1.12 .10^{-7}$ & $3.27 .10^{-7}$ \\
\hline$(0.0,0.5)$ & $3.19 .10^{-5}$ & $4.69 .10^{-5}$ & $6.78 .10^{-7}$ & $6.64 .10^{-7}$ \\
\hline$(1,0.6)$ & $3.18 .10^{-5}$ & $4.89 .10^{-5}$ & $4.50 .10^{-7}$ & $3.36 .10^{-7}$ \\
\hline$(1.5,0.7)$ & $1.95 .10^{-5}$ & $1.79 .10^{-5}$ & $7.07 .10^{-7}$ & $1.79 .10^{-6}$ \\
\hline$(2,0.8)$ & $1.78 .10^{-5}$ & $3.18 .10^{-5}$ & $4.29 .10^{-7}$ & $1.02 .10^{-6}$ \\
\hline$(2.5,0.9)$ & $4.91 .10^{-5}$ & $3.27 .10^{-5}$ & $1.02 .10^{-6}$ & $5.17 .10^{-7}$ \\
\hline$(3,1.0)$ & $8.08 .10^{-5}$ & $2.02 .10^{-5}$ & $1.41 .10^{-6}$ & $6.44 .10^{-7}$ \\
\hline
\end{tabular}

Table 4: Maximum absolute errors for problem (27).

\begin{tabular}{|c|c|c|}
\hline$N$ & $M_{1}$ & $M_{2}$ \\
\hline 4 & $6.51 \times 10^{-1}$ & $8.65 \times 10^{-1}$ \\
8 & $1.63 \times 10^{-3}$ & $1.83 \times 10^{-3}$ \\
12 & $6.43 \times 10^{-7}$ & $1.69 \times 10^{-6}$ \\
\hline
\end{tabular}

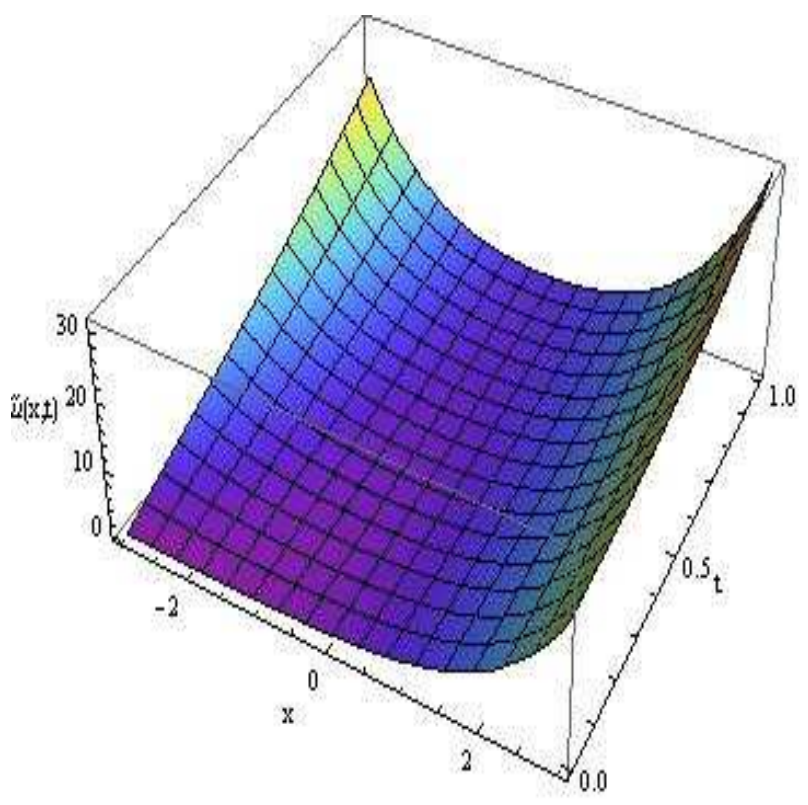

Fig. 9: The approximate solution $\widetilde{u}(x, t)$ for example 2 at $N=14$.

The exact solutions of Eqs. (27) are

$u(y, t)=e^{y} \cosh (t)+e^{-y} \sinh (t), \quad v(y, t)=e^{-y} \cosh (t)-e^{y} \sinh (t)$.

Absolute errors of $u(y, t)$ and $v(y, t)$ related to (27)-(29) are introduced in Table 3 using C-GR-C method with two choices of $N$ in the interval $[-3,3]$. Moreover, maximum absolute errors of $u(y, t)$ and $v(y, t)$ related to (22)-(24) are introduced in Table 4 using C-GL-C method with various choices of $N$.

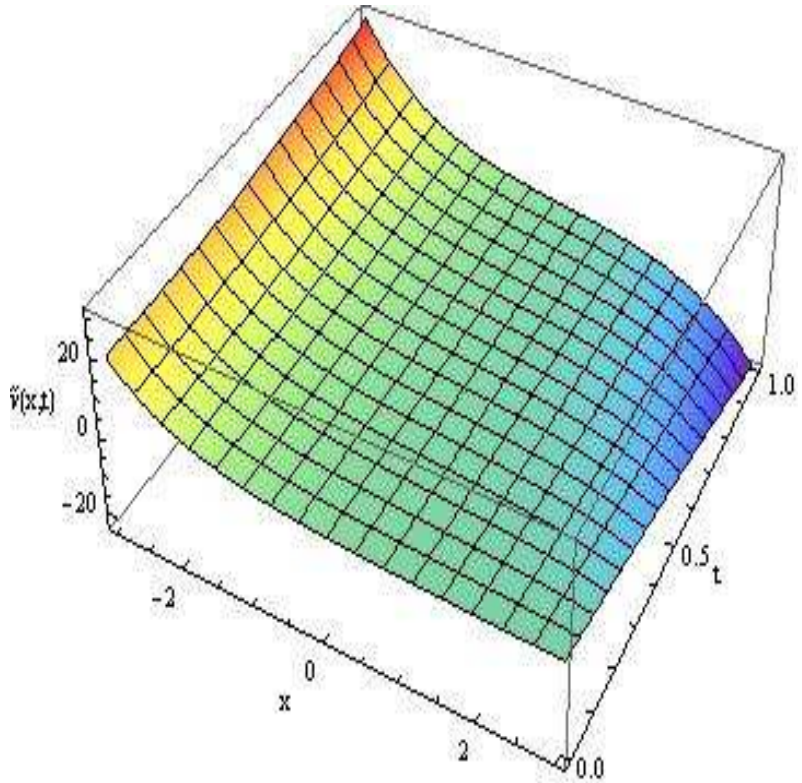

Fig. 10: The approximate solution $\widetilde{v}(x, t)$ for example 2 at $N=$ 14.

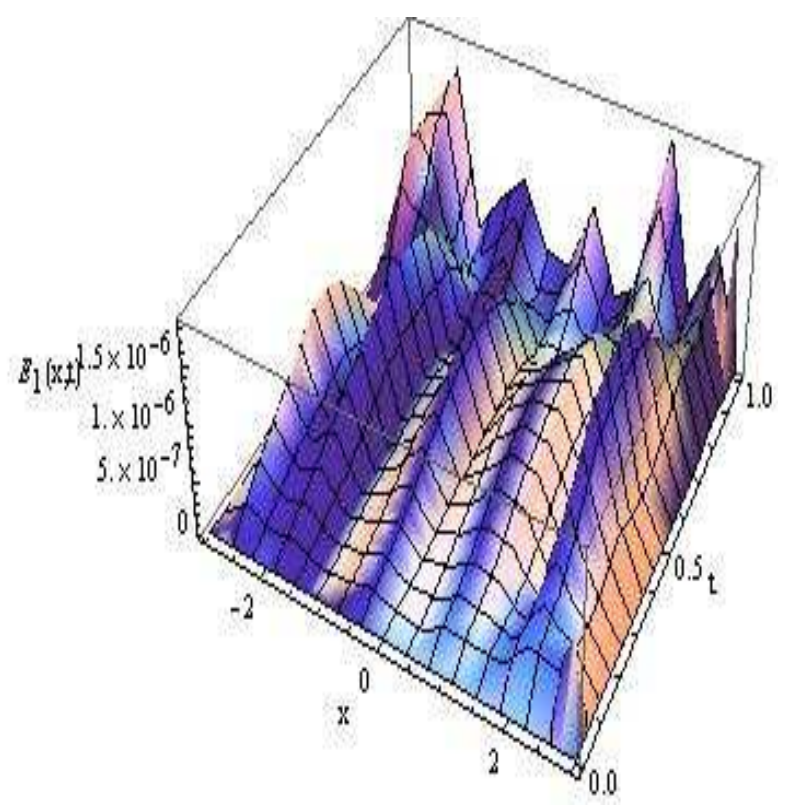

Fig. 11: The absolute error $E_{1}(x, t)$ for example 2 at $N=14$.

The approximate solutions $\widetilde{u}(x, t)$ and $\widetilde{v}(x, t)$ of problem (27) at $N=12$ are displayed in Figs. 9 and 10, respectively. While, in Figs. 11 and 12, we present the absolute errors $E_{1}(x, t)$ and $E_{2}(x, t)$ at $N=12$. Moreover, we plotted the approximate solutions $\widetilde{u}(x, t)$ and $\widetilde{v}(x, t)$, and the exact solutions $u(x, t)$ and $v(x, t)$ in Figs. 13, 14, 15 and 16, respectively, with values of parameters listed in their captions. Also wee see that the approximate and 


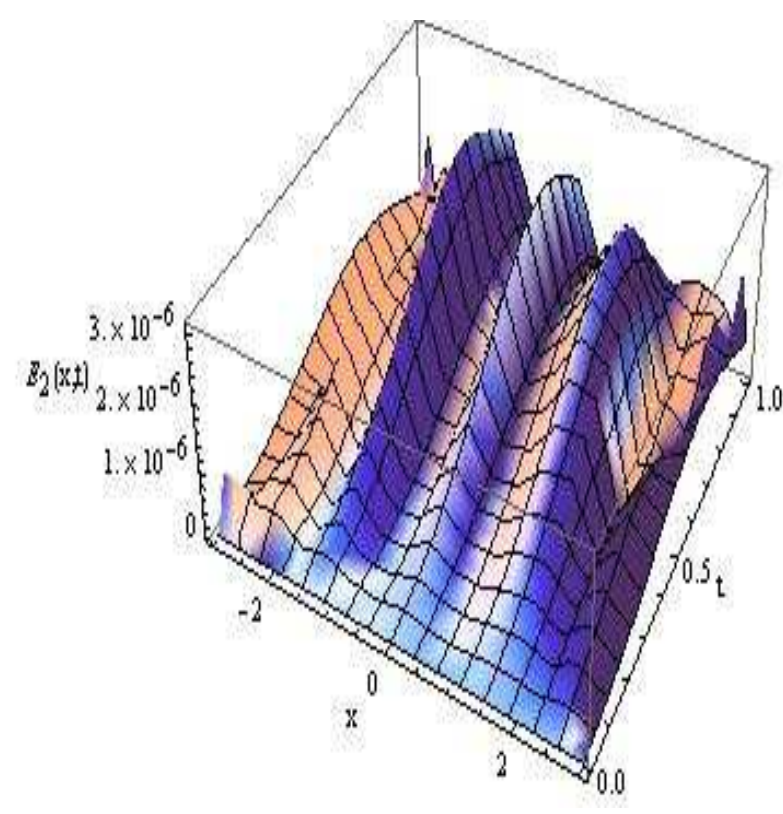

Fig. 12: The absolute error $E_{2}(x, t)$ for example 2 at $N=14$.

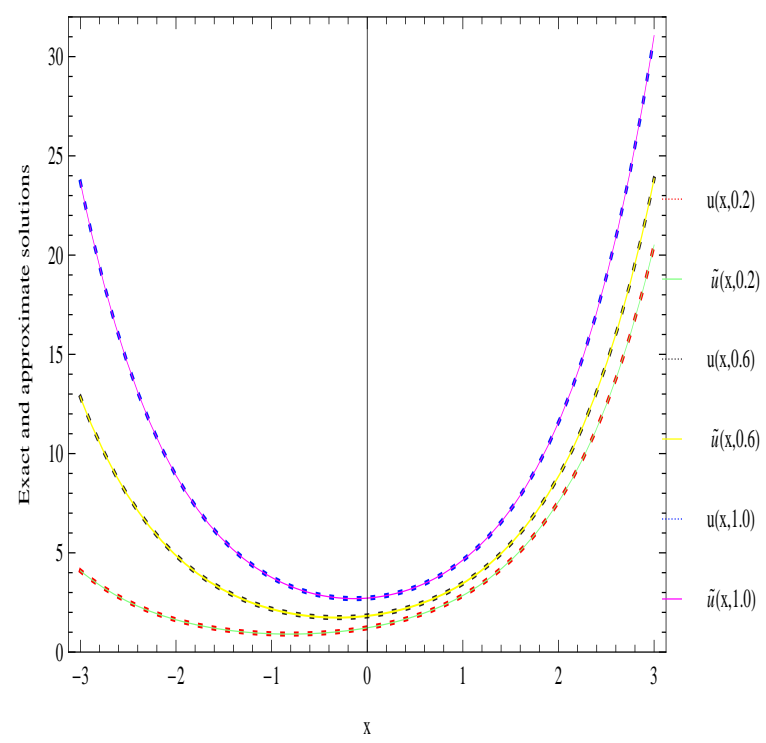

Fig. 13: The approximate solution $\widetilde{u}(x, t)$ and the exact solution $u(x, t)$ for $t=0.2,0.6$ and 1.0 for example 2 at $N=14$.

the exact solutions are coincided for different values of $t$ or $x$.

\section{Conclusion}

In this paper, we have proposed a numerical algorithm to solve the nonlinear hyperbolic system of first order using

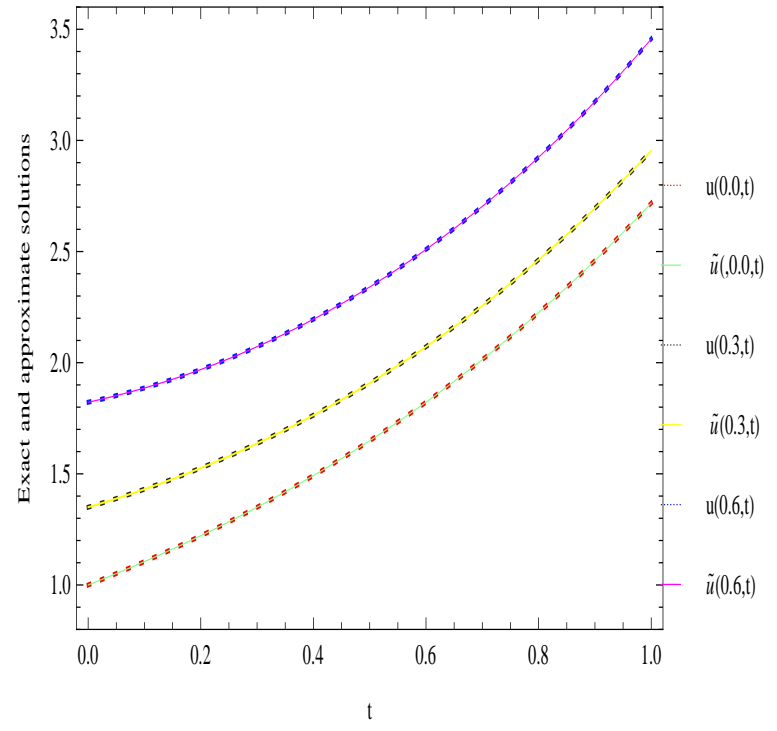

Fig. 14: The approximate solution $\widetilde{u}(x, t)$ and the exact solution $u(x, t)$ for $x=0.0,0.3$ and 0.6 for example 2 at $N=14$.

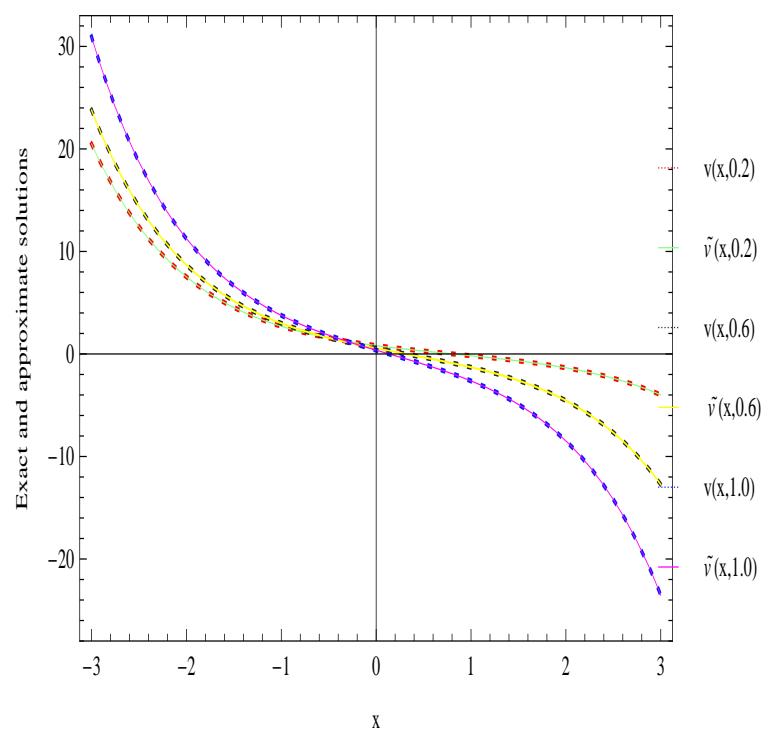

Fig. 15: The approximate solution $\widetilde{v}(x, t)$ and the exact solution $v(x, t)$ for $t=0.2,0.6$ and 1.0 for example 2 at $N=14$.

Gauss-Radau-collocation points and approximating directly the solution using the Chebyshev polynomials. The numerical results given in the previous section demonstrate the good accuracy of this algorithm. Moreover, The algorithm introduced in this paper can be well suited for handling general linear and nonlinear partial differential equations with initial-boundary 


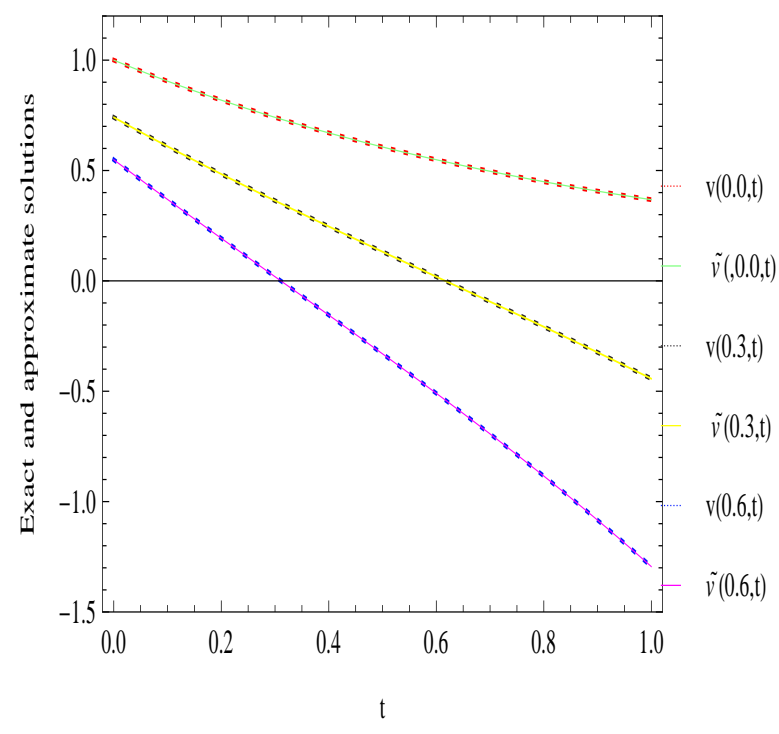

Fig. 16: The approximate solution $\widetilde{v}(x, t)$ and the exact solution $v(x, t)$ for $x=0.0,0.3$ and 0.6 for example 2 at $N=14$.

conditions. We note that the algorithm presented here can be applied based on Legendre, Jacobi polynomials or others polynomials to general linear and nonlinear partial differential systems.

\section{References}

[1] A. Guaily, M. Epstein, Mechanics Research Communications, 37, 158-163 (2010).

[2] G. A. Pinter, Nonlinear Analysis, 53, 653-668 (2003).

[3] Q. Ma, C. Zhong, Applied Mathematics and Computation, 157, 745-758 (2004).

[4] D. D. Joseph, M. Renardy, J-C. Saut, Archive for Rational Mechanics and Analysis, 87, 213-251 (1985).

[5] M. Dehghan, International Journal of Computer Mathematics, 83, 123-129 (2006).

[6] S. Larsson, F. Saedpanah, IMA Journal of Numerical Analysis, 30, 964-986 (2010).

[7] M. Wysocki, Mathematics and Computers in Simulation, 25, 335-345 (1983).

[8] R. Lhner, K. Morgan, O. C. Zienkiewicz, International Journal for Numerical Methods in Fluids, 4, 1043-1063 (1984).

[9] R. S. Falk, G. R. Richter, Lecture Notes in Computational Science and Engineering, 11, 209-219 (2000).

[10] T. J. R. Hughes, T. E. Tezduyar, Computer Methods in Applied Mechanics and Engineering, 45, 217-284 (1984).

[11] G. M. Hulbert, T. J. R. Hughes, Computer Methods in Applied Mechanics and Engineering, 84, 327-348 (1990).

[12] J. A. Rossmanith, Journal of Computational Physics, 213, 629-658 (2006).

[13] E. Bertolazzi, G. Manzini, Mathematics and Computers in Simulation, 75, 141-160 (2007).
[14] M. Dumbser, M. Käser, Journal of Computational Physics, 221, 693-723 (2007).

[15] H. Kim, R. Sakthivel, Communications in Nonlinear Science and Numerical Simulation, 17, 3788-3794 (2012).

[16] P. Pedram, M. Mirzaei, S.S. Gousheh, Computer Physics Communications, 176, 581-588 (2007).

[17] Y. Sun, Y.C. Zhou, S-G. Li, G. W. Wei, Journal of Computational Physics, 214, 466-490 (2006).

[18] J-G. Tang, H-P. Ma, Applied Numerical Mathematics, 57, 1-11 (2007).

[19] P. K. Dutt, S. Bedekar, Journal of Computational and Applied Mathematics, 134, 165-190 (2001).

[20] D. Gottlieb, J. S. Hesthaven, Journal of Computational and Applied Mathematics, 128, 83-131 (2001).

[21] R. Sakthivela, C. Chuna, A-R. Bae, International Journal of Computer Mathematics, 87, 2601-2606 (2010).

[22] C. Chun, R. Sakthivel, Computer Physics Communications, 181, 1021-1024 (2010).

[23] B-Y. Guo, X-Y. Zhang, Applied Numerical Mathematics, 57, 455-471 (2007).

[24] A. H. Bhrawy, Applied Mathematics and Computation http://dx.doi.org/10.1016/j.amc.2013.07.056, (2013).

[25] Z. Jackiewicz, B. Zubik-Kowal, Applied Numerical Mathematics, 56, 433-443 (2006).

[26] S-L. Chang, H-S. Chen, B-W. Jeng, C-S. Chien, Journal of Computational and Applied Mathematics, 254, 2-16 (2013).

[27] Y. Chen, T. Tang, Journal of Computational and Applied Mathematics, 233, 938-950 (2009).

[28] A. Rahmoune, Applied Mathematics and Computation, 219, 9254-9260 (2013).

[29] K. Zhang, J. Li, H. Song, Applied Mathematics and Computation, 218, 10848-10860 (2012).

[30] G. N. Elnagar, M. Kazemi, Journal of Computational and Applied Mathematics, 76, 147-158 (1996).

[31] T. A. Driscoll, Journal of Computational Physics, 229, 5980-5998 (2010).

[32] Y-J. Jiang, Journal of Computational and Applied Mathematics, 230, 333-340 (2009).

[33] Y. Jiang, J. Ma, Journal of Computational and Applied Mathematics, 244, 115-124 (2013).

[34] F. Fakhar-Izadi, M. Dehghan, Journal of Computational and Applied Mathematics, 235, $4032-4046$ (2011).

[35] S. Nguyen, C. Delcarte, Journal of Computational Physics, 200, 34-49 (2004).

[36] C. Canuto, M. Y. Hussaini, A. Quarteroni, T. A. Zang, Spectral Methods: Fundamentals in Single Domains. Springer-Verlag, New York, (2006).

[37] C. I. Gheorghiu, Spectral Methods for Differential Problems, T. Popoviciu, Institute of Numerical Analysis, Cluj-Napoca, Romaina, (2007).

[38] R. G. Voigt, D. Gottlieb, M. Y. Hussaini, Spectral Methods for Partial Differential Equations, SIAM, Philadelphia, (1984).

[39] E. H. Doha, A. H. Bhrawy, M. A. Saker, Applied Mathematics Letters, 24, 559-565 (2011)

[40] X. Xiong, X. Zhao, J. Wang, Numerical Algorithms, 63, 691-711 (2013)

[41] A. H. Bhrawy, S. I. El-Soubhy, Applied Mathematics and Computation, 217, 2684-2697 (2010). 
[42] S. N. Fata, L. J. Gray, Computational Mechanics, 44, 263271 (2009).

[43] E. H. Doha, A. H. Bhrawy, R. M. Hafez, Communications in Nonlinear Science and Numerical Simulation, 17, 38023810 (2012).

[44] S. R. Lau, R. H. Price, Journal of Computational Physics, 231, 7695-7714 (2012).

[45] F. Ghoreishi, S. Yazdani, Computers \& Mathematics with Applications, 61, 30-43 (2011).

[46] A. Rahmoune, Applied Mathematics and Computation, 219, 9254-9260 (2013).

[47] Y-S. Sun, B-W. Li, Journal of Quantitative Spectroscopy and Radiative Transfer, 113, 2205-2212 (2012).

[48] J. Hagan, Journal of Computational Physics, 238, 210-216 (2013).

[49] A. H. Bhrawy and M. A. Alghamdi, Boundary Value Problems, doi:10.1186/1687-2770-2012-62, (2012)

[50] L. Zhu and Q. Fan, Communications in Nonlinear Science and Numerical Simulation, 17, 2333-2341 (2012).

[51] E. H. Doha, A. H. Bhrawy and S. S. Ezz-Eldien, Applied Mathematical Modelling, 36, 4931-4943 (2012).

[52] A. Akyüz, M. Seze, Applied Mathematics and Computation, 144, 237-247 (2003).

[53] E. H. Doha, A. H. Bhrawy, S. S. Ezz-Eldien, Applied Mathematical Modelling, 35, 5662-5672 (2011).

[54] M. Dehghan, A. Taleei, Computer Physics Communications, 182, 2519-2529 (2011).

[55] V. G. Panchang, D. A. Kopriva, Mathematical and Computer Modelling, 12, 625-640 (1989).

[56] A. Pinelli, C. Benocci, Mi. Deville, Computer Methods in Applied Mechanics and Engineering, 116, 201-210 (1994).

[57] A. Akyüz-Dascioglu, Applied Mathematics and Computation, 151, 221-232 (2004).

[58] E. Babolian, S. Abbasbandy, F. Fattahzadeh, Applied Mathematics and Computation, 198, 35-43 (2008).

[59] M. Gülsu, Y. öztürk, M. Sezer, Applied Mathematics and Computation, 216, 2183-2198 (2010).

[60] A. Akyüz-Dascioglu, M. Sezer, Journal of the Franklin Institute, 342, 688-701 (2005).

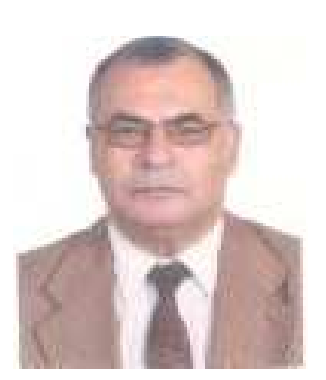

Eid H. Doha, Ph.D., is Professor of Mathematics (Numerical analysis and approximation theory) at university of Cairo, Egypt. He has published over 70 research papers in different areas of mathematics, including special functions, orthogonal polynomials, difference equations, spectral solutions of ordinary, partial, and fractional differential equations, boundary-value problems, initial-value problems, wavelets, and free and moving boundary-value problems. His current research interest includes connection and linearization problems between continuous, discrete, and orthogonal polynomials. Spectral methods for ordinary, partial, and fractional differential equations.

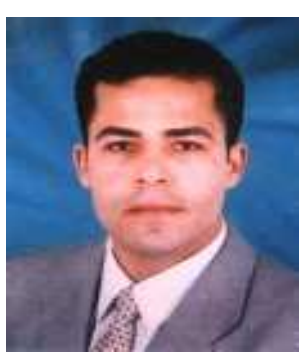

Ali H. Bhrawy is Associate Professor of Mathematics (Numerical analysis and scientific computing) at King Abdulaziz University, Jeddah, Saudi Arabia. His main research interests are: Spectral methods and their applications in partial differential equations, fractional differential equations and delay Differential equations. Orthogonal polynomials, Bernstein polynomials and multi-degree reduction of Bezier curves on computer aided geometric design. Exact solutions for nonlinear partial differential equations.

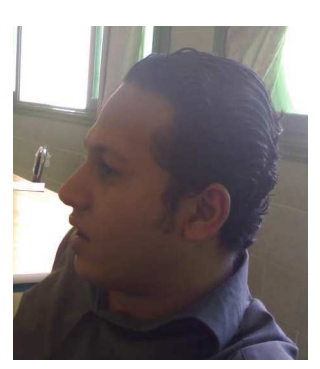

Ramy M. Hafez is Assistant Lecturer of Department of Basic Science, Institute of Information Technology, Modern Academy, Cairo, Egypt. His main research interests are: Numerical analysis and scientific computing; Spectral methods and their applications in linear and nonlinear partial differential equations, and nonlinear fractional multi-order differential equations.

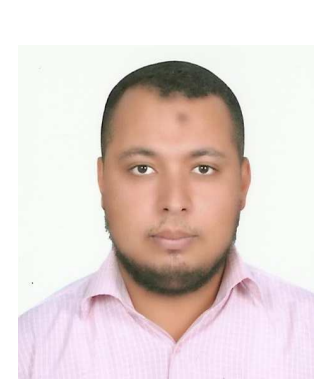

Mohamed A Abdelkawy is Assistant Lecturer of Mathematical Departement, Beni-Suef University, Beni-Suef, Eygpt. His main research interests are: Spectral methods for solving nonlinear ordinary, partial and fractional differential equations. Nonlinear phenomena which appears in many scientific and engineering fields. 